\title{
Prevalence and predicting factors of non-alcoholic fatty liver disease diagnosed by transient elastography with controlled attenuation parameter in HIV-infected patients
}

\section{Tanawat Jongraksak}

Mahidol University Faculty of Medicine Ramathibodi Hospital

Abhasnee Sobhonslidsuk ( $\nabla$ abhasnee.sob@mahidol.ac.th )

Division of Gastroenterology and Hepatology, Department of Medicine, Faculty of Medicine, Ramathibodi hospital, Mahidol University https://orcid.org/0000-0002-3730-5532 Janjira Jatchavala

Mahidol University Faculty of Medicine Ramathibodi Hospital

Daruneewan Warodomwichit

Mahidol University Faculty of Medicine Ramathibodi Hospital

Piyaporn Kaewduang

Mahidol University Faculty of Medicine Ramathibodi Hospital

Somnuek Sungkanuparph

Mahidol University Faculty of Medicine Ramathibodi Hospital

Research article

Keywords: Non-alcoholic fatty liver disease; Human immune deficiency virus; Transient elastography; Controlled attenuation parameters; Liver stiffness

Posted Date: August 30th, 2019

DOl: https://doi.org/10.21203/rs.2.11887/v1

License: (c) (i) This work is licensed under a Creative Commons Attribution 4.0 International License. Read Full License 


\section{Abstract}

Background: HIV infection is one of the most important health problems worldwide. With effective antiretroviral therapy (ART), the mortality rate of HIV-infected patients has significantly declined. Currently, metabolic complications become more common and lead to increasing morbidity and mortality in HIV-infected patients. Non-alcoholic fatty liver disease (NAFLD) has emerged as a common metabolic complication in these patients. Our study was aimed to determine the prevalence and predicting factors of NAFLD in HIV-infected population.

Methods: A cross-sectional study was conducted in HIV-infected patients receiving ART without history of regular alcohol consumption or known chronic liver diseases. The data of demographic and clinical parameters, metabolic profiles, bioelectrical impedance analysis, liver ultrasonography and transient elastography (TE) with controlled attenuation parameter (CAP) were collected. The prevalence and predicting factors of NAFLD were analyzed. NAFLD was defined when CAP $\geq 248 \mathrm{~dB} / \mathrm{m}$. Patients were categorized into two groups: NAFLD and non-NAFLD. Significant liver fibrosis was defined when liver stiffness measurement (LSM) $>7.0 \mathrm{kPa}$. Predicting factors of NAFLD were determined using logistic regression analysis.

Results: A total of 150 patients were included in the study. Forty-eight (32.0\%) patients had NAFLD. Among this group, significant fibrosis was diagnosed in 5 (10.4\%) patients and steatosis by ultrasonography was documented in 31 (64.6\%) patients. Baseline characteristics including age, sex, CD4 cell count, types of ART regimens and duration of ART between the two groups were not different $(p>0.05)$. Blood pressure, waist circumference, body mass index (BMI), percentage of fat, AST, ALT, $\mathrm{HbA1c}, \mathrm{LDL}$, and triglycerides were higher in the NAFLD group $(\mathrm{p}<0.05)$. By multivariate analysis, high BMI [odds ratio (OR) 1.596; 95\% confidence interval (CI), 1.336-1.907, $p<0.001$ ], triglycerides level more than $150 \mathrm{mg} / \mathrm{dl}$ [OR 3.722; $95 \% \mathrm{Cl}, 1.508-9.187, \mathrm{p}<0.004$ ] and advance age [OR $1.076 ; 95 \% \mathrm{Cl}, 1.017-$ $1.139, p<0.011]$ were predicting factors of NAFLD.

Conclusions: NAFLD is commonly found in HIV-infected patients receiving ART while significant fibrosis is relatively uncommon. High BMI, age and triglycerides appear to be predicting factors of NAFLD. The regimen and duration of ART are not associated with NAFLD. Screening for NAFLD by TE with CAP in HIVinfected patients should be recommended for early detection of NAFLD.

\section{Background}

HIV infection is a common and important health problem worldwide. Life expectancy of HIV-infected patients has significantly increased due to effective antiretroviral therapy (ART). In 2017, the magnitude of HIV infection in adults was 36.9 million cases globally. Among this number, 21.7 million was on ART [1]. Currently, metabolic complications become more common and lead to high morbidity and mortality in HIV-infected patients [2-4]. Non-alcoholic fatty liver disease (NAFLD) has emerged as a common 
metabolic complication in HIV-infected patients. There is a strong association between NAFLD and metabolic syndrome. HIV infection is probably one of the risk factors of NAFLD [5].

NAFLD is the most common liver disease in developed countries [6]. It was defined when liver fat accumulation exceeding $5 \%$ of the liver in the absence of regular alcohol consumption [6]. Clinical presentation of NAFLD could be asymptomatic or mild increased serum transaminases. Furthermore, hepatocellular carcinoma can occur in NAFLD without the background of liver cirrhosis [7]. Liver biopsy has been the gold standard procedure to diagnose NAFLD but it is invasive and unrealistic in clinical practice especially in HIV-infected patients [6]. Currently, non-invasive tools have replaced liver biopsy for liver fibrosis assessment. Transient elastography (TE) with controlled attenuation parameter (CAP) and liver ultrasonography can be used as screening tools for NAFLD in HIV infected patients [8-10]. A strong correlation of CAP and fat accumulation in the liver has been reported in clinical studies [8, 11]. CAP has been reported to be useful in diagnosis of liver steatosis [8].

There are only a few studies that determine the prevalence and predicting factors of NAFLD in HIVinfected population [12-16]. HIV infected patients have higher risk of metabolic syndrome compared to normal population. Early detection of NAFLD in HIV-infected patients with appropriate intervention may prevent the progression of NAFLD and the development of complications. The primary objective of this study was to evaluate prevalence in HIV-infected patients. The secondary objectives were to determine the predicting factors of NAFLD and severity of NAFLD in HIV-infected patients.

\section{Methods}

\section{Patients}

A cross-sectional study was conducted at Ramathibodi Hospital, Bangkok, Thailand during October 2017 to October 2018. HIV-infected patients who were on ART with complete virological suppression (undetectable HIV RNA) and without history of alcohol consumption were enrolled to the study from the Infectious Disease Clinic.

The main inclusion criteria were HIV-infected patients who were receiving ART with undetectable HIV viral load within 6 months and gave signed consent to participate in the study. The exclusion criteria included patients with the presence of viral hepatitis B or hepatitis $\mathrm{C}$ co-infection, other known liver disease such as cirrhosis or hepatocellular carcinoma, critical illness or active opportunistic infection (OI), and active alcohol consumption (male $>30 \mathrm{~g} /$ day, female $>20 \mathrm{~g} /$ day) [16]. Our protocol was reviewed and approved by the Committee of Human Rights Related to Research Involving Human Subjects, Faculty of Medicine Ramathibodi Hospital, Mahidol University, and the study was conducted in accordance with the principle of Good Clinical Practice.

Baseline characteristic, anthropometric measurement, bioelectrical impedance analysis and biochemical data 
General information such as age, sex, ART regimen, history of OI, underlying condition, current medications were reviewed from medical database. Blood pressure and metabolic profiles including body weight $(\mathrm{BW})$, height $(\mathrm{Ht})$, waist circumference $(\mathrm{WC})$ and hip circumference $(\mathrm{HC})$ were collected. Body mass index (BMI) were calculated. Biological impedance analysis was done in all patients for muscle mass, fat mass, percentage of fat, basal metabolic rate (BMR), appendicular skeletal muscle mass $\left(\mathrm{ASM} / \mathrm{Ht}^{2}\right.$ ). Blood tests including complete blood count, CD4 cell count, HIV RNA, blood urea nitrogen, creatinine, liver function test, fasting plasma glucose (FPG), 2-hour 75-gram oral glucose tolerance test (OGTT), insulin level, lipid profiles, hepatitis $B$ surface antigen and anti-hepatitis $C$ antibody were performed. The diagnosis of pre-diabetes and diabetes were based on the definition from American Diabetes Association in 2017 [17]. The study protocol is shown in Figure 1.

\section{Assessment of fatty liver and liver stiffness by transient elastography with controlled attenuation parameter (CAP) and ultrasonography}

TE with CAP and liver ultrasonography were performed on the same day and after 6 -hour fasting. The probe transducer was placed on the skin, between the ribs at the level of the right lobe of liver by a single experienced operator who performed 10 valid acquisitions with interquartile range/median (IQR/M) of liver stiffness (LS) less than $0.3[18,19]$. The software then calculated the median values of LS and CAP. In this study, NAFLD was defined as CAP at least $248 \mathrm{~dB} / \mathrm{m}$ [20]. The cut-off values at 248, 268 and 280 $\mathrm{dB} / \mathrm{m}$ were used to define NAFLD stage $\mathrm{S} 1$ (steatosis $>5 \%$ ), NAFLD stage $\mathrm{S} 2$ (steatosis $>33 \%$ ) and NAFLD stage S3 (steatosis $>66 \%$ ) [20]. Significant liver fibrosis was defined as LS at least 7.1 [16]. Liver cirrhosis was defined as LS at least $13 \mathrm{kPa}$ with the presence of cirrhotic features from ultrasonography $[16,21$, 22].

Liver ultrasonography was performed and the result was interpreted by a single experienced hepatobiliary radiologist. Fatty liver was identified when liver echogenicity exceeded that of renal cortex and spleen and there was attenuation of ultrasound wave, loss of definition of diaphragm and poor delineation of the intrahepatic architecture [9]. The degree of fatty liver by ultrasonography was classified to mild, moderate, and severe hepatic steatosis [9].

\section{Non-invasive fibrosis markers}

The simple biomarker fibrosis-4 index (FIB-4) was also calculated as follows: (age (years) $x$ aspartate aminotransferase (AST))/(platelet count $\left(10^{9} / \mathrm{I}\right) \times$ (alanine aminotransferase (ALT) $)^{0.5}$ [23]. A standard cut-off value of significant fibrosis was 2.67 [24]. AST to platelet ratio index (APRI) was calculated and it was correlated with the severity of liver fibrosis [16]. APRI score greater than 1.0 had a sensitivity of $76 \%$ and a specificity of $72 \%$ for predicting of cirrhosis [16]. NAFLD fibrosis score (NFS) was calculated to estimate the amount of scarring in liver in NAFLD group by using the cut-offs $<-1.455$ as F0-F2, $-1.455-$ 0.675 as indeterminate score and $>0.675$ as F3-4. NFS was calculated from nafldscore.com $[23,25,26]$. 


\section{Statistical analysis}

We estimated the sample size for each group using the n4studies program version 1.4.0 [27]. The sample size was calculated on the basis of a previous study that revealed the prevalence of NAFLD at $48 \%$ [16]. Assuming a power of $80 \%$ and an alpha of 0.05 , we needed a sample of at least 131 individuals in a twotailed test. We compared the characteristics of participants between groups by outcome status using Student's $\mathrm{t}$ test or Mann-Whitney U test when appropriate for independent continuous variables. Chisquare test was used to compare any independent categorical values between groups. Predicting factors of NAFLD were determined using logistic regression analysis. Final models were adjusted for tentative predicting factors. Results were reported as odds ratio (OR) with $95 \%$ confidence interval (Cl). All tests were two-tailed and with a significance level at 0.05 . The difference was considered statistically significant when $P$ value was less than 0.05 . Statistical analyses were performed using SPSS statistic version 23.

\section{Results}

\section{Patient characteristics}

A total of $150 \mathrm{HIV}$-infected patients were included in this study after meeting the inclusion and exclusion criteria. Demographic data and other baseline characteristics are summarized in Table 1. Of all, 48 patients had NAFLD by CAP criteria, accounting for a prevalence of $32 \%$ in this HIV-infected study population.

\section{Comparison of baseline characteristics and HIV profiles between the NAFLD and non-NAFLD groups}

Among 150 HIV-infected patients, $55.30 \%$ were male with mean age \pm standard deviation of $45.83 \pm 9.54$ years. NAFLD patients were older with the greater number of male and diabetes (Table 1). Mean body weight, BMI, WC, WC/HC ratio, systolic blood pressure and diastolic blood pressure in the NAFLD group were significantly higher than the non-NAFLD group. From biological impedance analysis, mean muscle mass, fat mass, percentage of fat and $A S M / \mathrm{Ht}^{2}$ of the NAFLD group were significantly higher than the non-NAFLD group, which was corresponded with a higher BMR in the NAFLD group.

The median (range) duration of ART was 7.40 (4.40-8.90) years (Table 2). One hundred and ten (73.3\%) patients were on non-nucleoside reverse transcriptase inhibitor (NNRTI)-based regimens with 32 $(21.3 \%)$ patients on protease inhibitor $(\mathrm{PI})$-based regimens, and 8 (5.3\%) patients on other regimens such as integrase inhibitor-based regimen. The pattern of ART regimen use was not significantly different between the two groups.

\section{Laboratory data}

Mean hemoglobin, AST, ALT and gamma-glutamyl transferase, total protein and creatinine were significantly higher in the NAFLD group than the non-NAFLD group (Table 3). The NAFLD group had 
triglycerides (TG), low-density lipoprotein cholesterol (LDL), FPG, 2-hr OGTT and hemoglobin A1c (HbA1c) significantly higher than the non-NAFLD group. Median (IQR) insulin was higher in NAFLD group. In addition, patients with NAFLD also had significantly lower level of high-density lipoprotein cholesterol (HDL).

\section{Non-invasive hepatic steatosis and fibrosis assessment}

From the examination of the 48 patients in the NAFLD group by TE, 15 (31.3\%) patients were in $\mathrm{S} 1$ steatosis; 8 (16.7\%) patients were in S2 steatosis; and 25 (52.1\%) patients were in S3 steatosis (Table 4). Of all, 50 (33.3\%) patients had steatosis from ultrasonography and $31(64.6 \%)$ patients were in the NAFLD group. Among these 50 patients, 37 (24.7\%) patients had mild steatosis, 11 (7.3\%) patients had moderate steatosis and $2(1.3 \%)$ patients had severe steatosis. CAP significantly correlated with the degree of steatosis from ultrasonography with a Spearman Rank correlation coefficient of $0.42(p<0.003)$ (Figure 2).

Among patients with NAFLD, 5 (10.4\%) patents had significant fibrosis from LS criteria. Two patients who were diagnosed cirrhosis from ultrasonography were referred to the liver clinic for further investigation. The mean APRI score, but not the mean FIB-4 and NFS scores, was significantly higher in the NAFLD than the non-NAFLD groups. The mean NFS in the NAFLD group was $-2.12 \pm 1.21$, which signified the absence of significant fibrosis $[23,25,26]$.

\section{Predicting factors of NAFLD by US and CAP criteria in HIV-infected patients}

From univariate analysis, sex, age, BMI, systolic blood pressure, hemoglobin, percentage of CD4 cell, creatinine, ALT, HDL, TG, $\mathrm{HbA1c}$, percentage of fat and $\mathrm{ASM} / \mathrm{Ht}^{2}$ by biological impedance analysis were associated with NAFLD in this study population. From multivariate analysis, high BMI [odds ratio (OR) $1.596 ; 95 \%$ confidence interval $(\mathrm{Cl}), 1.336-1.907, p<0.001]$, triglyceride level more than $150 \mathrm{mg} / \mathrm{dL}$ [OR $3.722 ; 95 \% \mathrm{Cl}, 1.508-9.187, \mathrm{p}<0.004$ ] and advance age [OR 1.076; $95 \% \mathrm{Cl}, 1.017-1.139, \mathrm{p}<0.011]$ were independent predicting factors of NAFLD in HIV population, as shown in Table 5.

\section{Discussion}

The prevalence of NAFLD in HIV-infected patients who were on ART and without the history of viral hepatitis infection, heavy alcohol drinking or other known chronic liver disease was $32 \%$, which was comparable to the results from the previous studies (35\%-55\%) [12, 14-16, 28, 29]. A recent meta-analysis study of 10 moderate to low quality articles using different methods to assess hepatic steatosis demonstrated that the prevalence of NAFLD was $35 \%(95 \% \mathrm{Cl}, 29-42)$ [14]. CAP at least $248 \mathrm{~dB} / \mathrm{m}$ was used to define NAFLD in this study, similarly to the previous studies $[15,20]$. However, using various cutoffs values for NAFLD may lead to a wide range of prevalence of NAFLD in HIV-infected population $[16,28,30]$. 
In this study, the prevalence of significant liver fibrosis was $10.4 \%$ among HIV-infected patients with NAFLD, which was lower than the results from the previous reports $(15 \%-22 \%)[12,14,16]$. The lower rate of significant liver fibrosis may be related to the fewer number of patients with PI-based regimen use in this study. From a previous study, patients who were on PI had increased risk of significant fibrosis [16]. PI may increase the risk of hyperlipidemia and diabetes by suppressing proteasome-mediated breakdown of nascent apolipoproteins B, resulting in the overproduction of TG-rich lipoproteins and an increased risk of insulin resistant by inhibiting glucose transporter 4 at adipose and muscle [31]. Therefore, PI may increase the risk of liver steatosis and significant fibrosis. From this hypothesis, the duration of ART should increase the risk of metabolic syndrome and NAFLD. However, the duration of ART was not found to be a predicting factor for NAFLD in this study.

To the best of our knowledge, our report is the first study to explore the prevalence of NAFLD in HIVinfected patients in South-east Asia. Nevertheless, we acknowledge several limitations of this study. Although TE with CAP and liver ultrasonography are non-invasive tools often used for assessing NAFLD in clinics, they are not gold standard diagnostic tools. Liver biopsy, a gold standard histological assessment, was not done to confirm the diagnosis of NAFLD. There were some cases with diagnostic discrepancy between the two tests. However, the accuracy of CAP in comparison with liver biopsy for the evaluation of liver steatosis has been reported [20]. A recent meta-analysis study showed that the cut-off value of CAP at $248 \mathrm{~dB} / \mathrm{m}$ had the sensitivity and specificity of $69.0 \%$ and $82 \%$ to detect NAFLD stage S1 (i.e. liver steatosis $>5 \%$ ) [20]. In addition, CAP values were correlated with degree of hepatic steatosis from ultrasonography (Figure 2). In the settings that TE with CAP is not available, ultrasonography may be used to detect hepatic steatosis with at least a moderate degree of liver fat deposit. This study is a crosssectional study from a single center that provides the standard care for HIV-infected patients.

Longitudinal study should be conducted for assessing the relationship between HIV infection and the development of hepatic steatosis [32].

We also carried out biological impedance analysis to study body component of the study patients. We suspected that HIV-infected patients may have sarcopenic obesity with low muscle mass in patients with normal or high body weight. In contrast, the study results demonstrated the presence of copious muscle mass correlating with increased body weight [33]. This could be due to a good standard care of HIV patients.

From the results of our study, the independent predictors of NAFLD were high BMI, advance age, and serum TG level higher than $150 \mathrm{mg} / \mathrm{dL}$ which are similar to the results from the previous studies [12,1416 , 28]. Screening for the presence of NAFLD in HIV patients who have these factors is useful for early detection of NAFLD in these patients.

\section{Conclusion}

NAFLD is commonly found in HIV-infected patients receiving ART. Nevertheless, significant fibrosis is relatively infrequent in the HIV infected population. High BMI, advance age and serum TG level above 150 
$\mathrm{mg} / \mathrm{dL}$ appear to be the predicting factors of NAFLD. Types of ART regimen and duration of ART taking are not associated with the presence of NAFLD. Screening for NAFLD by TE with CAP in HIV-infected patients with these predicting factors should be recommended for early detection of NAFLD.

\section{Abbreviations}

ART: Antiretroviral therapy; HIV: Human immunodeficiency virus; NAFLD: Non-alcoholic fatty liver disease; CAP: Controlled attenuation parameter; TE: Transient elastography; OI: Opportunistic infection; BW: Body weight; Ht: Height; WC: Weight circumference; HC: Hip circumference; BMR: Basal metabolic rate; ASM/Ht2: Appendicular skeletal muscle mass; IQR/M: Interquartile range/median; NNRTI: Non-nucleoside reverse transcriptase inhibitor; PI: Protease inhibitor; AST: Aspartate aminotransferase; ALT: Alanine aminotransferase; TG: Triglyceride; FBG: Fasting blood glucose; HbA1c: Hemoglobin A1c; HDL: Highdensity lipoprotein cholesterol; LDL: Low-density lipoprotein cholesterol; FIB-4: Fibrosis-4 index; APRI: AST to platelet ratio index; NFS: NAFLD fibrosis score; OR: Odds ratio; Cl: Confidence interval.

\section{Declarations}

\section{Ethics approval and consent to participate}

The study protocol was approved by the Committee on Human Rights related to Research Involving Human Subjects, Faculty of Medicine, Ramathibodi Hospital (ID 08-60-02). It was conducted according to the Good Clinical Practice Guideline after obtaining informed consent. Informed consent obtained from the study participants as stated in the method part.

\section{Consent for publication}

Not applicable

\section{Availability of data and materials}

The dataset used and/or analyzed during the current study is available from the corresponding author upon request.

\section{Competing interests}

The authors declare that they have no competing interest. 


\section{Funding}

This study was funded by Faculty of Medicine Ramathibodi Hospital, Mahidol University and the Thailand Research Fund (Prof. Somnuek Sungkanuparph). They did not have roles in the study design and data collection, data analysis, data analysis or the manuscript writing.

\section{Authors' contributions}

TJ participated in the design of the study, data collection, analysed and interpreted the data, and drafted the manuscript. AS contributed to the design of the study, interpreted the data, reviewed and edited the manuscript. JJ, DW and PK participated in data collection and involved in drafting the manuscript. SS participated in the design of the study, analyzed and interpreted the data, drafted and reviewed the manuscript. All authors gave final approval of the final version of the manuscript to be published, and all are agree to be accountable to all aspects of the work.

\section{Acknowledgements}

We are grateful for the participation of all study patients and support from all attending staff and research nurses of the Division of Infectious Diseases and Division of Gastroenterology of Hepatology of the Department of Medicine, and Department of Radiology.

\section{Author details}

${ }^{1}$ Department of Medicine, Faculty of Medicine, Ramathibodi Hospital, Mahidol University, Bangkok, Thailand.

${ }^{2}$ Department of Radiology, Faculty of Medicine, Ramathibodi Hospital, Mahidol University, Bangkok, Thailand.

${ }^{3}$ Chakri Naruebodindra Medical Institute, Faculty of Medicine Ramathibodi Hospital, Mahidol University, Bangkok, Thailand.

\section{References}

1. HIV and AIDS in Thailand. http://www.unaids.org/en/regionscountries/countries/thailand. 2. Jerico C, Knobel H, Montero M, Ordonez-Llanos J, Guelar A, Gimeno JL et al: Metabolic syndrome among HIVinfected patients: prevalence, characteristics, and related factors. Diabetes Care 2005;28:132-137. 3. Wand H, Calmy A, Carey DL, Samaras K, Carr A, Law MG et al: Metabolic syndrome, cardiovascular disease and type 2 diabetes mellitus after initiation of antiretroviral therapy in HIV infection. Aids 
2007;21:2445-2453. 4. Manosuthi W, Chottanapand S, Thongyen S, Chaovavanich A, Sungkanuparph S: Survival rate and risk factors of mortality among HIV/tuberculosis-coinfected patients with and without antiretroviral therapy. J Acquir Immune Defic Syndr 2006;43:42-46. 5. Vallet-Pichard A, Mallet V, Pol S: Nonalcoholic fatty liver disease and HIV infection. Semin Liver Dis 2012;32:158-166. 6. Chalasani N, Younossi Z, Lavine JE, Diehl AM, Brunt EM, Cusi K et al: The diagnosis and management of non-alcoholic fatty liver disease: practice Guideline by the American Association for the Study of Liver Diseases, American College of Gastroenterology, and the American Gastroenterological Association. Hepatology 2012;55:2005-2023. 7. Michelotti GA, Machado MV, Diehl AM: NAFLD, NASH and liver cancer. Nat Rev Gastroenterol Hepatol 2013;10:656-665. 8. Mikolasevic I, Orlic L, Franjic N, Hauser G, Stimac D, Milic S: Transient elastography (FibroScan $((\mathrm{R}))$ ) with controlled attenuation parameter in the assessment of liver steatosis and fibrosis in patients with nonalcoholic fatty liver disease - Where do we stand? World $\mathrm{J}$ Gastroenterol 2016;22:7236-7251. 9. Hernaez R, Lazo M, Bonekamp S, Kamel I, Brancati FL, Guallar E et al: Diagnostic accuracy and reliability of ultrasonography for the detection of fatty liver: a meta-analysis. Hepatology 2011;54:1082-1090. 10. Matta B, Lee TH, Patel K: Use of Non-invasive Testing to Stage Liver Fibrosis in Patients with HIV. Curr HIV/AIDS Rep 2016;13:279-288. 11. Lemoine M, Assoumou L, De Wit S, Girard PM, Valantin MA, Katlama C et al: Diagnostic Accuracy of Noninvasive Markers of Steatosis, $\mathrm{NASH}$, and Liver Fibrosis in HIV-Monoinfected Individuals at Risk of Nonalcoholic Fatty Liver Disease (NAFLD): Results From the ECHAM Study. J Acquir Immune Defic Syndr 2019;80:e86-e94. 12. Lombardi R, Sambatakou H, Mariolis I, Cokkinos D, Papatheodoridis GV, Tsochatzis EA: Prevalence and predictors of liver steatosis and fibrosis in unselected patients with HIV mono-infection. Dig Liver Dis 2016;48:14711477. 13. Lui G, Wong VW, Wong GL, Chu WC, Wong CK, Yung IM et al: Liver fibrosis and fatty liver in Asian HIV-infected patients. Aliment Pharmacol Ther 2016;44:411-421. 14. Maurice JB, Patel A, Scott AJ, Patel K, Thursz M, Lemoine M: Prevalence and risk factors of nonalcoholic fatty liver disease in HIVmonoinfection. Aids 2017;31:1621-1632. 15. Perazzo H, Cardoso SW, Yanavich C, Nunes EP, Morata M, Gorni $\mathrm{N}$ et al: Predictive factors associated with liver fibrosis and steatosis by transient elastography in patients with HIV mono-infection under long-term combined antiretroviral therapy. J Int AIDS Soc 2018;21:e25201. 16. Vuille-Lessard E, Lebouche B, Lennox L, Routy JP, Costiniuk CT, Pexos C et al: Nonalcoholic fatty liver disease diagnosed by transient elastography with controlled attenuation parameter in unselected HIV monoinfected patients. Aids 2016;30:2635-2643. 17. Chamberlain JJ, Herman WH, Leal S, Rhinehart AS, Shubrook JH, Skolnik N et al: Pharmacologic Therapy for Type 2 Diabetes: Synopsis of the 2017 American Diabetes Association Standards of Medical Care in Diabetes. Ann Intern Med 2017;166:572-578. 18. Boursier J, Zarski JP, de Ledinghen V, Rousselet MC, Sturm N, Lebail B et al: Determination of reliability criteria for liver stiffness evaluation by transient elastography. Hepatology 2013;57:1182-1191. 19. Castera L, Friedrich-Rust M, Loomba R: Noninvasive Assessment of Liver Disease in Patients With Nonalcoholic Fatty Liver Disease. Gastroenterology 2019;156:12641281.e1264. 20. Karlas T, Petroff D, Sasso M, Fan JG, Mi YQ, de Ledinghen V et al: Individual patient data meta-analysis of controlled attenuation parameter (CAP) technology for assessing steatosis. J Hepatol 2017;66:1022-1030. 21. Wong VW, Vergniol J, Wong GL, Foucher J, Chan HL, Le Bail B et al: Diagnosis of fibrosis and cirrhosis using liver stiffness measurement in nonalcoholic fatty liver disease. Hepatology 2010;51:454-462. 22. Roulot D, Costes JL, Buyck JF, Warzocha U, Gambier N, Czernichow S et al: 
Transient elastography as a screening tool for liver fibrosis and cirrhosis in a community-based population aged over 45 years. Gut 2011;60:977-984. 23. Shah AG, Lydecker A, Murray K, Tetri BN, Contos MJ, Sanyal AJ: Comparison of noninvasive markers of fibrosis in patients with nonalcoholic fatty liver disease. Clin Gastroenterol Hepatol 2009;7:1104-1112. 24. 2. Classification and Diagnosis of Diabetes. Diabetes Care 2017;40:S11-s24. 25. Angulo P, Hui JM, Marchesini G, Bugianesi E, George J, Farrell GC et al: The NAFLD fibrosis score: a noninvasive system that identifies liver fibrosis in patients with NAFLD. Hepatology 2007;45:846-854. 26. Treeprasertsuk S, Bjornsson E, Enders F, Suwanwalaikorn S, Lindor KD: NAFLD fibrosis score: a prognostic predictor for mortality and liver complications among NAFLD patients. World J Gastroenterol 2013;19:1219-1229. 27. n4Studies. 1.4.0 ed. 28. Macias J, Gonzalez J, Tural C, Ortega-Gonzalez E, Pulido F, Rubio R et al: Prevalence and factors associated with liver steatosis as measured by transient elastography with controlled attenuation parameter in HIV-infected patients. Aids 2014;28:1279-1287. 29. Sulyok M, Makara M, Rupnik Z, Ferenci T, Ujhelyi E, Kormos L et al: Hepatic steatosis in individuals living with HIV measured by controlled attenuation parameter: a cross-sectional study. Eur J Gastroenterol Hepatol 2015;27:679-685. 30. Macias J, Real LM, Rivero-Juarez A, Merchante $\mathrm{N}$, Camacho A, Neukam K et al: Changes in liver steatosis evaluated by transient elastography with the controlled attenuation parameter in HIV-infected patients. HIV Med 2016;17:766-773. 31. Echevarria KL, Hardin TC, Smith JA: Hyperlipidemia associated with protease inhibitor therapy. Ann Pharmacother 1999;33:859-863. 32. Pembroke T, Deschenes M, Lebouche B, Benmassaoud A, Sewitch M, Ghali P et al: Hepatic steatosis progresses faster in HIV mono-infected than HIV/HCV co-infected patients and is associated with liver fibrosis. J Hepatol 2017;67:801-808. 33. van Welzen BJ, Mudrikova T, El Idrissi A, Hoepelman AIM, Arends JE: A Review of Non-Alcoholic Fatty Liver Disease in HIV-Infected Patients: The Next Big Thing? Infect Dis Ther 2019;8:33-50.

\section{Legends}

Figure 1. Protocol flow chart

Figure 2. Correlation between controlled attenuation parameter (CAP) and grading of hepatic steatosis from ultrasonography

Table 1. Baseline characteristics and bioelectrical impedance analysis between non-alcoholic fatty liver disease (NAFLD) and non-NAFLD groups

Table 2. History of anti-retroviral therapy (ART), opportunistic infection (OI) and family history of metabolic conditions between non-alcoholic fatty liver disease (NAFLD) and non-NAFLD groups. 
Table 3. Laboratory data between non-alcoholic fatty liver disease (NAFLD) and non-NAFLD groups.

Table 4. Non-invasive hepatic steatosis and fibrosis assessment between non-alcoholic fatty liver disease (NAFLD) and non-NAFLD groups.

Table 5. Univariate and multivariate analysis for the factor associated with the presence of non-alcoholic fatty liver disease (NAFLD).

\section{Tables}

Due to technical limitations, tables are only available as a download in the supplemental files section

\section{Figures}




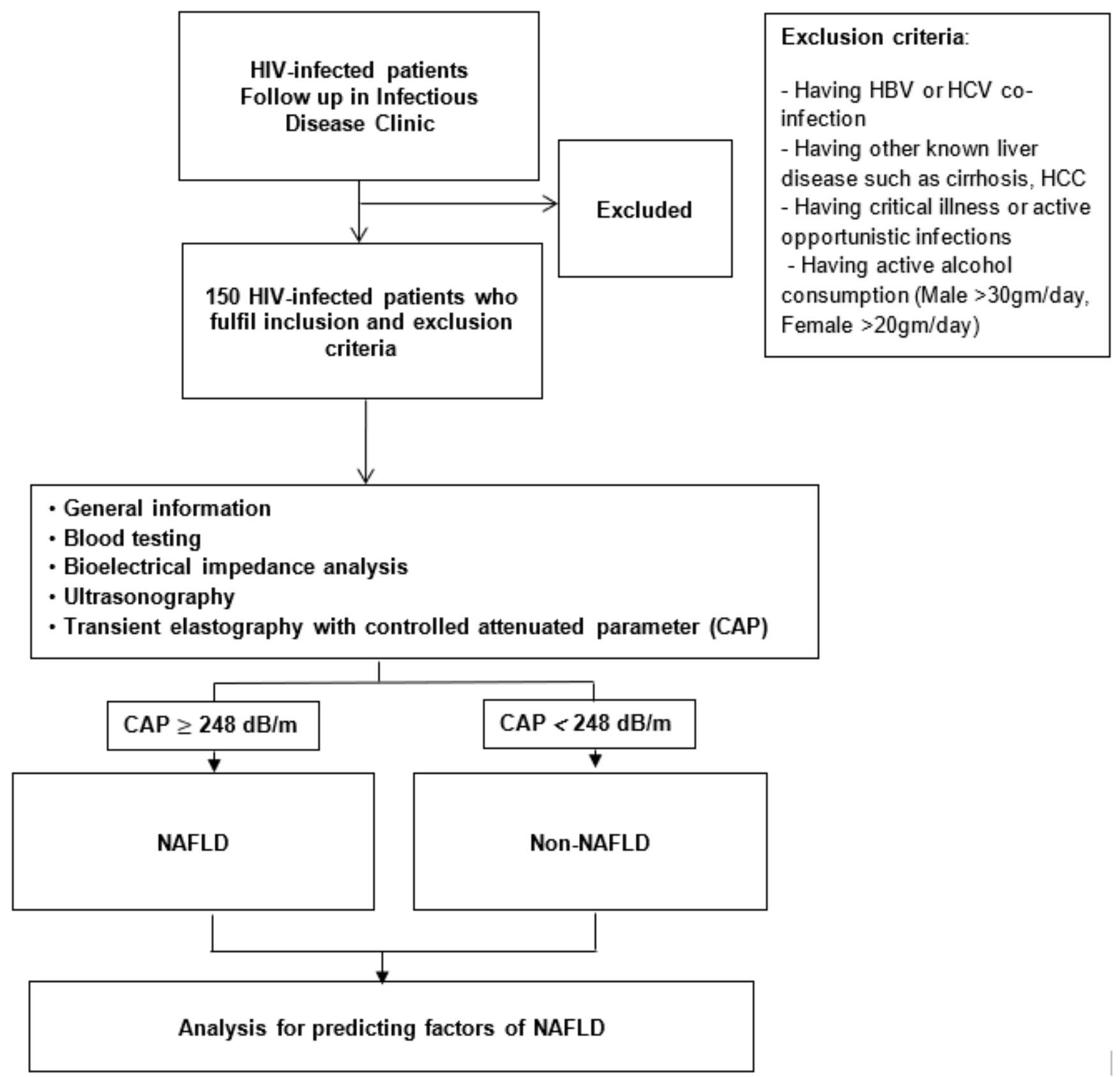

Figure 1

Protocol flow chart 


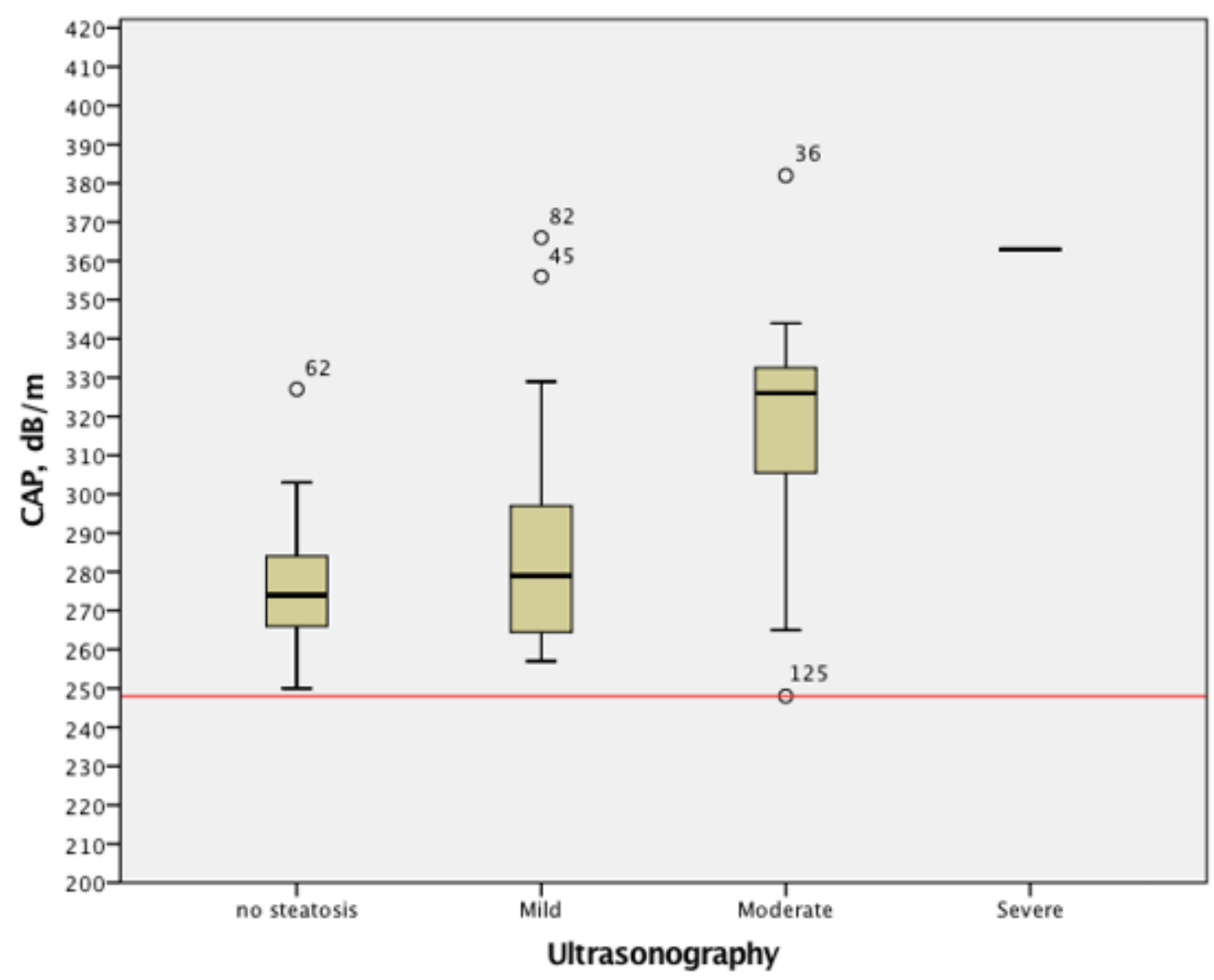

Figure 2

Correlation between controlled attenuation parameter (CAP) and grading of hepatic steatosis from ultrasonography

\section{Supplementary Files}

This is a list of supplementary files associated with this preprint. Click to download.

- TableNAFLD.pdf 